\title{
Néel Temperature of Antiferromagnets for Phase Transitions Driven by Spin-wave Interactions ${ }^{\dagger}$
}

\author{
Andrés Ayuela, ${ }^{\mathrm{a}, \mathrm{b}, *}$ Douglas J. Klein, ${ }^{\mathrm{c}}$ and Norman H. March ${ }^{\mathrm{b}, \mathrm{d}, \mathrm{e}}$

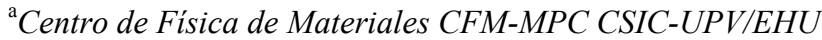 \\ ${ }^{\mathrm{b}}$ Donostia International Physics Center (DIPC), Departamento de Física de Materiales, Fac. de Químicas, \\ Univ. del Pais Vasco UPV-EHU, 20018 San Sebastián, Spain \\ ${ }^{\mathrm{c}}$ Marine Sciences, Texas A\&M University at Galveston, P.O. Box 1675, Galveston, Texas 77553, USA. \\ ${ }^{\mathrm{d}}$ Dept. of Physics, Univ. of Antwerp, Antwerp, Belgium. \\ ${ }^{\mathrm{e}}$ Oxford University, Oxford, England
}

RECEIVED MARCH 19, 2013; REVISED OCTOBER 18, 2013; ACCEPTED OCTOBER 18, 2013

\begin{abstract}
In a recent article, 1 a wide variety of phase transitions, with transition $(\mathrm{t})$ temperature $T_{\mathrm{t}}$, were shown to be usefully characterized by the form $k_{\mathrm{B}} T_{\mathrm{t}}=E_{\text {char }} \exp (-1 /|\lambda|)$ where $|\lambda|$ measured the strength of the quasiparticle interactions driving the phase transition. The present article is concerned primarily with antiferromagnets (AFs) having Néel temperature $T_{\mathrm{N}}$. It is first argued that the characteristic energy $E_{\text {char }}$ can be usefully represented by $k_{\mathrm{B}} \theta$, where $\theta$ is the Curie-Weiss temperature. This assertion is then confronted with experimental data on four insulating transition metal oxides, these being selected as all having the paramagnetic ions on a facecentered cubic lattice. For four of the five $\theta / T_{\mathrm{N}}$ is certainly greater than unity, the fifth having the ratio as unity to within experimental error. $|\lambda|$ is then argued to be related to physical parameters entering a mean field approximation to AFs. Finally, the above insulating Afs are complemented by a brief discussion of a metallic FeRh alloy where, in addition to having itinerant electrons and antiferromagnetism, elevating the temperature leads to a transition from an $\mathrm{AF}$ to a ferromagnetic state. (doi: $10.5562 / \mathrm{cca} 2266$ )
\end{abstract}

Keywords: antiferromagnets, transition metal oxides, Neél temperature, magnons, spin-waves

\section{INTRODUCTION}

In a recent work, ${ }^{1}$ one of us has been involved in treating a variety of phase transitions driven by quasiparticle interactions, including crystalline melting and the ferromagnetic-paramagnetic Curie point. One of us [AA] has been focusing on alloys and structural phases transitions with shape memory effect driven by magnetic fields. ${ }^{2-4}$ As such alloys were magnetic, more related studies of the ferromagnetic-paramagnetic transitions were made by looking at the spin spirals using first principles calculations. We found that crucial to a proper description of the transition was the softening of magnons for large values of reciprocal vectors. ${ }^{5}$ Our common papers ${ }^{6,7}$ using an Ising model were concerned with antiferromagnets and their transitions to paramagnetic(ferromagnetic) states. We found that it was also important to look at the staggered magnetization on the two sublattices. ${ }^{7}$

Here we deal specifically with the case of antiferromagnets around the Néel temperature $T_{\mathrm{N}}$, which we proposed should take the form $k_{\mathrm{B}} T_{\mathrm{N}}=E_{\text {char }} \exp (-1 /|\lambda|)$. The parameter $|\lambda|$ is taken to measure the strength of the spin-wave interactions. $E_{\text {char }}$ is argued to be given by $k_{\mathrm{B}} \theta$, where $\theta$ is the Curie-Weiss temperature and $k_{\mathrm{B}}$ is Boltzmann's constant. Some contact is established with known experimental properties of antiferromagnets, for four diatomic insulators where the paramagnetic ion lattice is fcc. Finally, the metallic alloy FeRh is briefly discussed and in particular, a temperature-induced transition from an antiferromagnetic to a ferromagnetic phase.

\section{FOUR INSULATING ANTIFERROMAGNETIC OXIDES WITH FCC STRUCTURE OF THE PAR- AMAGNETIC ION LATTICE}

Nagamiya,Yosida and $\mathrm{Kubo}^{8}$ record the experimental Néel temperature $T_{\mathrm{N}}$ for some 15 insulating antiferromagnets, and we summarize results for four of

\footnotetext{
$\dagger$ Dedicated to Professor Douglas Jay Klein on the occasion of his $70^{\text {th }}$ birthday.

* Author to whom correspondence should be addressed. (E-mail: swxayfea@sw.ehu.es)
} 
Table 1. Néel $T_{\mathrm{N}}$ and Curie-Weiss $\theta$ temperatures of some insulating antiferromagnets

\begin{tabular}{cccc}
\hline Material & $T_{\mathrm{N}} / \mathrm{K}$ & $\theta / \mathrm{K}$ & $\frac{\theta}{T_{\mathrm{N}}}$ \\
\hline $\mathrm{MnO}$ & 116 & 610 & 5.3 \\
$\mathrm{FeO}$ & 198 & 570 & 3.3 \\
$\mathrm{CoO}$ & 291 & 330 & 1.14 \\
$\mathrm{NiO}$ & 525 & $\approx 2000$ & $\approx 4$ \\
\hline
\end{tabular}

the prototype oxides materials in Table 1. The common feature of the four selected examples is that all have an fcc paramagnetic ion lattice. Recent works on these oxides $^{9}$ have also confirmed these Néel temperatures, while the Curie-Weiss temperature $\theta$ showed a larger scattering between various experimental measurements. This is also listed, again from experiment, in Table 1, together with the ratio $\theta / T_{\mathrm{N}}$.

These oxides have a basic superexchange mechanism between the local magnetic moments of $3 \mathrm{~d}$ traditional magnets, mediated by oxygen ions, similar to GdO ${ }^{10}$ This mechanism in these oxides is sustained by strong localization, but it is competing with other direct exchanges based on hybridization with band formation, as seen in ${ }^{11}$ and references therein.

Theories for the Néel temperatures in such compounds have to take into account the spin fluctuations and the change in the local magnetic moments. Within standard density functional theory, an improvement in the descriptions of correlations is needed in order to describe the temperature dependence of the localized magnetic moments. Sometimes as in $\mathrm{NiO}$ their survival is strongly dependent on the treatment of correlations. Such theories based on density functional theory study the fluctuations directly with ${ }^{12,13}$ or without ${ }^{14}$ quantum fluctuations. It is noteworthy that through these fluctuations at the transitions, local magnetic moments remain non-negligible above the transition temperature. Of course, the remanence of local moments is guaranteed using either the previous ${ }^{12-14}$ or Hartree-Fock ${ }^{11}$ results or the mappings onto Heisenberg models for the temperature description. Furthermore, good experimental and theoretical agreement is found for the Néel temperatures of $\mathrm{MnO}, \mathrm{FeO}$, and $\mathrm{CoO}$, except for $\mathrm{NiO}$, for which better correlation schemes or dynamical correlations including temperature dependence seem to be needed. As the controversy about these points has not yet been settled at high temperatures, not even the validity of the Heisenberg-like models used, ${ }^{15}$ we shall herein develop another approach that should describe the temperature dependence of antiferromagnets with just two sublattices, following ideas going back essentially to Weiss.

\section{AN EXPRESSION FOR NÉEL TEMPERATURES}

We begin by comparing the statistical quantities of fluids and magnetic systems, following table 3.5.1 in the book of Chaikin and Lubensky. ${ }^{16}$ For fluids, we have

$$
\frac{\partial\langle n\rangle}{\partial \mu}=\left\langle n^{2}\right\rangle \kappa_{T}=\beta S_{n n}
$$

where $n$ is the number of particles, $\mu$ is the chemical potential, $\kappa_{T}$ is the isothermal compressibility, $\beta=1 /\left(k_{\mathrm{B}} T\right)$, and $S_{n n}$ is the long wavelength $q$ limit of the liquid structure factor (see also ${ }^{1}$ ). This expression (Eq. 1) has its analogue for the magnetic susceptibility $\chi$

$$
\chi_{i j}=\frac{\partial\left\langle m_{i}\right\rangle}{\partial h_{j}}=\lim _{q \rightarrow 0} \beta G_{i j}(q)
$$

where $m_{i}$ and $h_{j}$ denote respectively the magnetization and the field in the $i$ and $j$ directions, while $G_{i j}$ is the order-parameter correlation function. In melts, we have

$$
k_{\mathrm{B}} T_{m}=E_{\text {char }} S_{T_{m}}(0)
$$

where $E_{\text {char }}$ is $\Omega / \kappa_{T}$ with $\Omega$ the atomic volume and $S_{T_{m}}(0)$ given by $0.03 \exp (-1 / \lambda)$. In solid-liquid transitions, the parameter $\lambda$ is related to thermal expansion in the solid, and it is determined in fact by the Grüneisen constant. ${ }^{1}$

Below, with the assumption that the phase transition at temperature $T_{\mathrm{N}}$ is driven by the interaction between anti-ferromagnons (AFM),we shall follow ${ }^{1}$ in writing

$$
k_{\mathrm{B}} T_{\mathrm{N}}=E_{\text {char }} \exp (-1 /|\lambda|)
$$

where $|\lambda|$ measures the strength of the AFM interactions. With respect to Ref. 1 we have that our Eq. 4 is the same as Eq. (16) in that reference with $\gamma_{q p}$ being replaced by $1 / \lambda$. This equation links the transition temperature with the number of magnons. The temperature values are related to a characteristic energy $E_{\text {char }}$. For example, when interaction among modes is negligible, $E_{\text {char }}$ will be the average energy of the available excited modes. Similarly to the Debye energy in phonons, $E_{\text {char }}$ is given by the Curie-Weiss constant in antiferromagnets. Also there is a dependence on the coupling between modes, given by $\lambda$. This coupling expresses both the energy between modes, like anharmonicity, and enters in the exponent of Eq. 4. It is noteworthy that in the limit of vanishing interaction, $\lambda=0$, i.e. the transition temperature goes to zero. However, in the limit of infinitely strong interaction, we have that $\lambda \rightarrow \infty$. The 
exponential becomes thus unity so that the transition temperature is the characteristic energy $E_{\text {char }}$.

The question of the nature of the characteristic energy $E_{\text {char }}$ is then, we believe, the major matter to settle for the four substances listed in Table 1. Early theoretical work summarized in Kittel's book ${ }^{17}$ (see Appendix) leads us to the proposal that the characteristic energy should be chosen as

$$
E_{\text {char }}=k_{\mathrm{B}} \theta
$$

where $\theta$ is the Curie-Weiss temperature, listed from experiment in Table 1 together with the ratio $\theta / T_{\mathrm{N}}$. Empirically support for the assumption (Eq. 5) applied via equation (Eq. 4) is then that in all materials in Table 1 the ratio $E_{\text {char }}=k_{\mathrm{B}} T_{\mathrm{N}}$ is greater than unity, as must result from equation (Eq. 4 ).

\section{Trends in Oxides of Late 3d Elements}

Then, it clearly follows that there must be a wide spread in the magnitude of the AFM interaction strength measured by $|\lambda|$, in $\mathrm{FeO}$ corresponding to $|\lambda| \approx 1$, while $\mathrm{NiO}$ and $\mathrm{MnO}$ have AFM strengths increasing from $\mathrm{FeO}$ in the above order. So $\mathrm{CoO}$ is the only example where one can conclude the strength is less than for FeO.

When comparing all the cases, we found that $\mathrm{MnO}$ and $\mathrm{NiO}$ behave similarly, while $\mathrm{FeO}$ and $\mathrm{CoO}$ depart from them. These differences can be explained using the results from previous calculations of the band structures for these oxides. ${ }^{11}$ On one hand, the oxides $\mathrm{MnO}$ and $\mathrm{NiO}$ behave similarly because they have half-full or full electronic shells. The basic magnetic mechanism of both compounds is thus superexchange. The difference of $\mathrm{NiO}$ with respect to $\mathrm{MnO}$ is that, as it has a full electronic shell, the treatment to deal with all the electrons will be largely sensitive to the chosen approach for the correlation. On the other hand, $\mathrm{FeO}$ and $\mathrm{CoO}$ have open shells, and other magnetic interactions, such as direct exchange, are competing with the superexchange. It seems that the stronger the hybridization with oxygen atoms, the closer is the ratio $\theta / T_{\mathrm{N}}$ to one.

\section{Meaning of the $|\lambda|$ Parameter}

At this point, however, we should note that in equation (Eq. 4), for the ferromagnetic-paramagnetic Curie temperature $T_{\mathrm{C}}$, it was argued that the spin-wave stiffness $D$ in the dispersion relation $\hbar \omega=D k^{2}$ in the long wavelength limit, determined $k_{\mathrm{B}} T_{\mathrm{C}}$ through $\sim D / a^{2}$ with $a$ the lattice parameter. We have not followed this, clearly appropriate, choice of $E_{\text {char }}$ in ferromagnets for the insulating antiferromagnetic listed in Table 1, since for these materials $\omega$ does not go to zero as $k \rightarrow 0$ because of the anisotropy field (see Figure 22 on page 344 of Kittel's book, for the spin-wave energy of AFM in tetragonal
$\mathrm{MnF}_{2}$ at $4.2 \mathrm{~K}$ observed by neutron inelastic scattering).

In magnetic systems we must also give some physical interpretation for the $\lambda$ similar to fluids which are driven by phonons as the interacting particles. We now have to rely on magnon-magnon interaction. Well known works ${ }^{18}$ have traditionally dealt with spin fluctuations, and they have divided these into transverse and longitudinal susceptibilities. The transverse modes are easily included in oxides, ${ }^{14}$ but this is not the case for the longitudinal ones. We feel that the $\lambda$ constant can be ascribed to how the longitudinal spin fluctuations depart from average values at $T=0$; in fact, they could end in spin canted solutions, unless locally and close to the transition. Such assignment compares well with the one given for fluids in terms of the Grüneisen parameter in the solid-liquid transitions.

At elevated temperatures and especially close to the transition temperature we must give up the image of non-interacting magnons. The magnons begin to interact strongly, and we must think more about strong spin fluctuations. Within a mean-field (MF) approach, they could be durable longitudinal oscillations, or beyond $\mathrm{MF}$ in the sense that coherent resonances happen in some parts of the sample. We anticipate that the first option is appropriate to describe the FM-AFM transitions while the second one describes probably better the transitions ending in a paramagnetic state.

\section{APPLICATION TO SOME AVAILABLE RE- SULTS ON METALLIC FERH IN AFM PHASE}

We next refer to the pioneering discussion of the metallic alloy FeRh as commented in the book on itinerant electron magnetism by Kübler. ${ }^{19}$ After Ref. 19, we found that the calculated free energies show a crossover from an antiferromagnetic state at low temperatures to a ferromagnetic phase. The temperature of the phase transition is predicted as $435 \mathrm{~K}$, whereas the experimental counterpart is $328 \mathrm{~K}$. Theory ${ }^{19}$ gives the magnetic moment of the Fe atoms as about $3 \mu_{\mathrm{B}}$. Symmetry, in fact, dictates that the magnetic moments of Rh vanish in the antiferromagnetic phase already referred to. We have thus a compound with Fe atoms separated by $\mathrm{Rh}$ atoms with zero moment. In principle, this compound can be thought as somewhat similar to the oxides except for the strong hybridization between the $\mathrm{Rh}$ and $\mathrm{Fe}$ atoms.

It is difficult to know whether such a transition is to be described by a formula such as proposed in equation Eq. 4 above, because this expression was designed to treat a 'simple' Néel temperature, in which the transition occurs to a phase with susceptibility of the CurieWeiss form: namely $\chi=$ constant $/(T+\theta)$. We feel this formula is more appropriate to describe the curve labeled $A M_{2}$ in Figure 5.13 of Kübler's book; this free energy curve intersects the paramagnetic phase at a 
temperature $\approx 750 \mathrm{~K}$, even though this transition is 'anticipated' by a crossover to ferromagnetism at $435 \mathrm{~K}$, as already mentioned above. Since the theoretical transition AFM-PM (not the experimental) is of first order, the similarity with the oxides is even reinforced. However, to keep the local magnetic moment through the second order transition as in oxides, the AFM-FM transition could also be considered but is beyond the scope of this letter.

In the review article ${ }^{9}$ we find the Néel and CurieWeiss temperatures recorded for alloys made of $\mathrm{FeRh}_{1-x} \mathrm{M}_{x}$ where $\mathrm{M}$ is a doping material around $\mathrm{Rh}$ in the periodic table. For very small values of $x$, the Mössbauer experiments show the characteristic signal of $\mathrm{Fe}$ in its original lattice. Thus, the $\mathrm{M}$ elements mostly exchange sites with the $\mathrm{Rh}$ atoms, and we can consider these compounds close to FeRh. The small doping $\mathrm{FeRh}_{0.85} \mathrm{Ir}_{0.15}$ expands slightly the lattice, but more important it results in the $T_{\mathrm{AFM}-\mathrm{FM}}$ being higher than $T_{\mathrm{N}}$ so that no ferromagnetic phase experimentally appears. In such measurements the Curie-Weiss law can be applied, and the authors find that the $\theta / T_{\mathrm{N}}$ ratio is close to 1 . This value is close to the one found for $\mathrm{CoO}$ which presents the strongest hybridization in previous oxides. It also agrees with the strong hybridization underlying the FeRh results commented on above.

This FeRh compound has been recently revisited by Sandratskii and Mavropoulos in Ref. ${ }^{20}$ Already in the AFM phase, before the FM state appears, they found that the local magnetic moment in Rh atoms must be remarkably different from zero. More remarkable, as the temperature increases, they show that within the AFM states, Fe local magnetic moments can adopt a non parallel situation in a canted configuration. The non-alignment of $\mathrm{Fe}$ atoms allows for $\mathrm{Rh}$ magnetization which indeed suffers longitudinal fluctuations. This finding corroborates, and is in perfect agreement with, the interpretation of the $|\lambda|$ parameter in previous sections.

\section{SUMMARY AND FUTURE DIRECTIONS}

For the insulating AFs in Table 1, we have argued phenomenologically that in equation (Eq. 4), which was used in 1 for ferromagnets with $E_{\text {char }}=D / a^{2}$, where $D$ measures the spin wave stiffness and $a$ is the lattice parameter, the AF case should take $E_{\text {char }} \sim k_{\mathrm{B}} \theta$, where $\theta$ is the Curie-Weiss temperature. However, $|\lambda|$ in equation (Eq. 4) continues to measure spin-wave interactions. These, we argue, are the quasiparticle interactions which drive the AF-paramagnetic transition in the insulating transition-metal oxides listed in Table 1. The main reason for the ferro- and AF-systems is that for the AF systems (compare $\mathrm{MnF}_{2}$ in Refs. ${ }^{17}$ ) there is the anisotropy field which can mean that $\mathrm{w}$ does not go to zero as $\mathrm{k} \rightarrow 0$. But $\lambda$ is still determined by magnon-magnon or spin-wave interactions.

We have also, but more briefly, selected one metallic alloy, FeRh, to compare and contrast with the insulating transition-metal oxides. One obvious difference is that the transition from the AF low temperature phase is now to a ferromagnetic state at a measured temperature of $328 \mathrm{~K}$. This is to be contrasted, in turn with the theoretically predicted value from itinerant electron calculations $^{19}$ of $435 \mathrm{~K}$. Symmetry ensures that in FeRh, the magnetic moments of $\mathrm{Rh}$ are zero in the $\mathrm{AF}$ phase. Hence, we argue for some similarity of this metallic alloy with the oxides in Table 1, except for the extensive hybridization between the metallic $\mathrm{Rh}$ and Fe atoms.

For the future, further calculations on FeRh seem called for, with particular attention to be paid to the transition temperature from the $\mathrm{AF}$ to the observed ferromagnetic phase. The itinerant electron magnetic treatment of FeRh recorded in Kübler's book ${ }^{19}$ is, of course, available to provide an excellent starting point for future refinements.

Acknowledgements. We acknowledge support from Basque Departamento de Educacion and the UPV/EHU (Grant No. IT366-07), the Spanish Ministerio de Educación y Ciencia (Grant o No. FIS2010-19609-CO2-02) and the ETORTEK program funded by the Basque Departamento de Industria and the Diputacion Foral de Guipúzcoa. NHM wishes to acknowledge that his contribution to this study was brought to fruition during a visit to DIPC, San Sebastián. He thanks Professors P.M. Echenique for continuing generous hospitality and much stimulation. Finally NHM acknowledges that his continuing affiliation with the University of Antwerp (UA) was made possible by the Professors D. Lamoev and C. V. Alsenoy through grant BOF-NOI (UA).

\section{APPENDIX}

\section{Néel Temperature $T_{\mathrm{N}}$ in Relation to the Curie-Weiss Temperature $\theta$}

As summarized by Kittel ${ }^{17}$ (see especially Eq. (40) in chapter 12), for $T>T_{\mathrm{N}}$ the experimental measurements of the susceptibility $\chi$ for the antiferromagnets in Table 1 take the form

$$
\chi=\frac{\text { constant }}{T+\theta}
$$

where $\theta$ is the Curie-Weiss temperature. Kittel notes that if a molecular field constant $-\varepsilon$, in the idea originally followed by Weiss, is introduced to describe interactions within a sublattice, then

$$
\frac{\theta}{T_{\mathrm{N}}}=\frac{\mu-\varepsilon}{\mu+\varepsilon}
$$


Kittel's equation (38) in the same chapter which reads

$$
T_{\mathrm{N}}=\mu C
$$

where $C$ refers to a single sublattice, such that $\mathrm{m}$ is about the interactions between sublattices, through the effective fields on the (assumed) two sublattices A and B take the form

$$
H_{\mathrm{A}}=H-\mu m_{\mathrm{B}}-\varepsilon m_{\mathrm{A}} \text { and } H_{\mathrm{B}}=H-\mu m_{\mathrm{A}}-\varepsilon m_{\mathrm{B}} .
$$

Problem 7 of Kittel in chapter 12 for four fcc sublattices explicitly gives $\theta / T_{\mathrm{N}}$. We shall next strengthen the previous deduction by discussing the mean field terms $\theta / T_{\mathrm{N}}=\frac{|\mu|+|\varepsilon|}{|\mu|-|\varepsilon|}$ due to the first and second neighbor interactions $|\mu|$ and $|\varepsilon|$ that drive the transition.

We begin with the Heisenberg hamiltonian, as given in,11 and following a mean field treatment. The transition Néel temperature is

$$
T_{\mathrm{N}}=\frac{S(S+1)}{3 k_{\mathrm{B}}} \sum_{l=1,2}\left(z^{\uparrow \uparrow}-z^{\uparrow \downarrow}\right) J_{l}
$$

where $z$ refers to the $z$-component of the spin $\left\langle s_{i}^{z}\right\rangle, S$ is the total spin, $\mathrm{kB}$ is the Boltzmann's constant, and $J_{l}$ is the exchange coupling that runs over first and second neighbors, used as cutoff for the hamiltonian. The $\mathrm{Cu}-$ rie-Weiss temperature that enters into the paramagnetic $\chi=C /(T+\theta)$ law is

$$
\theta=\frac{S(S+1)}{3 k_{\mathrm{B}}} \sum_{l=1,2}\left(z^{\uparrow \uparrow}+z^{\uparrow \downarrow}\right) J_{l}
$$

The value of $\theta$ depends on the total number of neighbors in each neighbor shell and it is independent of the magnetic ordering in a given crystal. As in the expansion we have $S(S+1)$, we are considering quantum fluctuations important for the exact determination of these temperatures. The ratio $\theta / T_{\mathrm{N}}$, however, is independent whether we consider classical or spin fluctuations. It depends via the $\mathrm{z}$ values on the local sublattices moments and via $J_{l}$ on their coupling which concerns both first and second neighbors. When com- paring with previous values of $\varepsilon$ and $\mu$ in Kittel's book, we found that they correspond to the sum of first and second neighbors of the products composed of $J_{l}$ couplings by local magnetic moments in configurations $\uparrow \uparrow$ and $\uparrow \downarrow$ respectively.

\section{REFERENCES}

1. N. H. March, E. V. Chulkov, P. M. Echenique, and C. C. Matthai, Phase Transitions (Taylor and Francis, UK) 83 (2010) 1086.

2. A. Ayuela, J. Enkovaara, K. Ullakko, and R. M. Nieminen, J. Phys.-Condens. Mat. 11 (1999) 2017.

3. A. Ayuela, J. Enkovaara, and R. M. Nieminen, J. Phys.-Condens. Mat. 14 (2002) 5325.

4. A. T. Zayak, P. Entel, J. Enkovaara, A. Ayuela, and R. M. Nieminen, J. Phys.-Condens. Mat. 15 (2003) 159; A. T. Zayak, P. Entel, J. Enkovaara, A. Ayuela, and R. M. Nieminen Phys. Rev. B 68 (2003) 132402.

5. J. Enkovaara, A. Ayuela, J. Jalkanen, L. Nordström, and R. M. Nieminen, Phys. Rev. B 67 (2003) 054417.

6. A. Misra, A. Ayuela, D. J. Klein, N. H. March, Phys. Lett. A 329 (2004) 396.

7. A. Ayuela, D. J. Klein, and N. H. March, Phys. Lett. A 362 (2007) 468 .

8. T. Nagamiya, K. Yosida, and R. Kubo, Advanc. Phys. 4 (1955) $1-112$.

9. S. Yuasa, Y. Otani, H. Miyajima, and A. Sakuma, IEEE Translation J. of Magnetics in Japan 9 (1994) 202, and references therein.

10. A. Ayuela, N. H. March, and D. J. Klein. J. Phys. Chem. A 111 (2007) 10162.

11. W. A. Harrison, Phys. Rev. B 77 (2008) 245103.

12. X. Ren, I. Leonov, G. Keller, M. Kollar, I. Nekrasov, and D. Vollhardt, Phys. Rev. B 74 (2006) 195114.

13. X. Wan, Q. Yin, and S. Y. Savrasov, Phys. Rev. Lett. 97 (2006) 266403.

14. I. D. Hughes, M. Däne, A. Ernst, W. Hergert, M. Lüders, J. B. Staunton, Z. Szotek, and W. M. Temmerman, New J. Phys. 10 (2008) 063010.

15. G. Fischer, M. Däne, A. Ernst, P. Bruno, M. Lüders, Z. Szotek, W. Temmerman, and W. Hergert, Phys. Rev. B 80 (2009) 014408 .

16. P. M. Chaikin and T. C. Lubensky, Principles of Condensed Matter Physics, Cambridge University Press, 1995.

17. C. Kittel, Introduction to Solid State Physics, John Wiley and Sons, Inc., New York, 2005.

18. G. G. Lonzarich and L. Taillefer, J. Phys. C Solid State 18 (1985) 4339

19. J. Kübler, Theory of Itinerant Electron Magnetism, Oxford University Press, 2000.

20. L. M. Sandratskii and P. Mavropoulos, Phys. Rev. B 83 (2011) 174408. 